\title{
A De Novo Mutation in TSC2 Gene is Associated with Tuberous Sclerosis Complex (TSC): A Case Report
}

\author{
Weihua Zhan', Jiangbo $\mathrm{Li}^{1}$, Yajie Zhao ${ }^{2}$, Jianli Zhang², Yaxi Zhang ${ }^{2}$, Yanxia $\mathrm{Mi}^{2}$ and Jianxiong Duan ${ }^{2 *}$ \\ ${ }^{1}$ Chengdu Shenkang Epilepsy Hospital, Chengdu 610000, People’s Republic of China
}

${ }^{2}$ Shanxi Guoxin Caregeno Medical Laboratory, Taiyuan 030006, People's Republic of China

*Corresponding author: Jianxiong Duan, Shanxi Guoxin Caregeno Medical Laboratory, Taiyuan 030006, People’s Republic of China, China

\begin{abstract}
Tuberous sclerosis, also known as tuberous sclerosis complex (TSC), is an autosomal dominant multisystem disorder characterized by hamartomas in multiple organ systems including brain, skin, heart, kidneys, and lung. These changes can result in epilepsy, learning difficulties, behavioral problems, renal failure, and other complications [1,2]. The affected genes are TSC1 and TSC2, encoding hamartin and tuberin respectively. The hamartin tuberin complex inhibits the mammalian target of rapamycin (mTOR) pathway, which controls cell growth and proliferation [2]. In this case, we report a 21-year-old Chinese female patient with TSC due to a de-nove mutation c.1806C > G (p. Tyr602Ter) in TSC2 gene [3]. This is likely to be the first report of this mutation in Chinese population. This mutation was evaluated as a pathogenic mutation based on the standards and guidelines of ACMG (American College of Medical Genetics and Genomics).
\end{abstract}

Keywords: Chinese; Epilepsy; Tuberous sclerosis complex (TSC); Tuberous sclerosis-1 (TSC1); Tuberous sclerosis-2 (TSC2); de novo mutation; ACMG

\section{Introduction}

TSC (tuberous sclerosis complex) is an autosomal dominant multi system disorder characterized by hamartomas in multiple organ systems. The genes which are related to TSC include two tumor-suppressor genes called TSC1 and TSC2. The TSC1 and TSC2 encoding hamartin and tuberin respectively. The hamartin tuberin complex inhibits the mammalian target of rapamycin (mTOR) pathway, which controls cell growth and proliferation [2]. Approximately 10 to $30 \%$ of cases of TSC are due to mutations in the TSC1 gene, the frequency of cases due to mutations in the TSC2 gene is consistently higher. TSC2 mutations are associated with more severe diseases [1]. The mutation of the gene TSC2 (c.1806C>G; p. Tyr602Ter) we detected does not exist in either Chinese population or any other populations. It has been identified as a pathogenic mutation in Clinvar database based on the hypotheses [4]. However, as of today, no detailed clinical study data or published research articles are found to support these hypotheses. It is likely to be the first clinical report of this mutation in the Chinese population.

\section{Case Report}

A21-year-old female patient who was born with no family history of seizures or other types of neurological disease has experienced Tuberous sclerosis complex (TSC) since she was 13-year-old. The patient experienced seizures seven to eight times per month. Most of the seizures occurred during the daytime and were presented as tonic-clonic seizures which lasted approximately 2 minutes in length. There was no obvious symptom prior to the seizures. But whenever the seizures occurred, the patient would experience severe fatigue over the whole body. Besides seizures, there were several other clinical features including mental retardation, a sebaceous adenoma and a brown-pigment patch on her forehead, many depigmentation spots of different sizes on her neck, chest, back and limbs. The clinical diagnosis of her seizures was epilepsy, but the exact cause of her seizures was not found. However, according to MRI scan which showed a nodular sclerosis at the parenchyma of her brain and various other diagnoses, tuberous sclerosis complex (TSC) was highly likely to be the cause of her epilepsy. As displayed 
in Figure 1A, the patient's Electroencephalography (EEG) showed frequent slow complex waves in the left anterior temporal area and the middle temporal area. Antiepileptic drugs of Carbamazepine,
Zolosamide, and Everolimus were prescribed, and the improvement of the syndromes of the patient was observed after one year, as the patient experienced less seizures (Figure 1B).

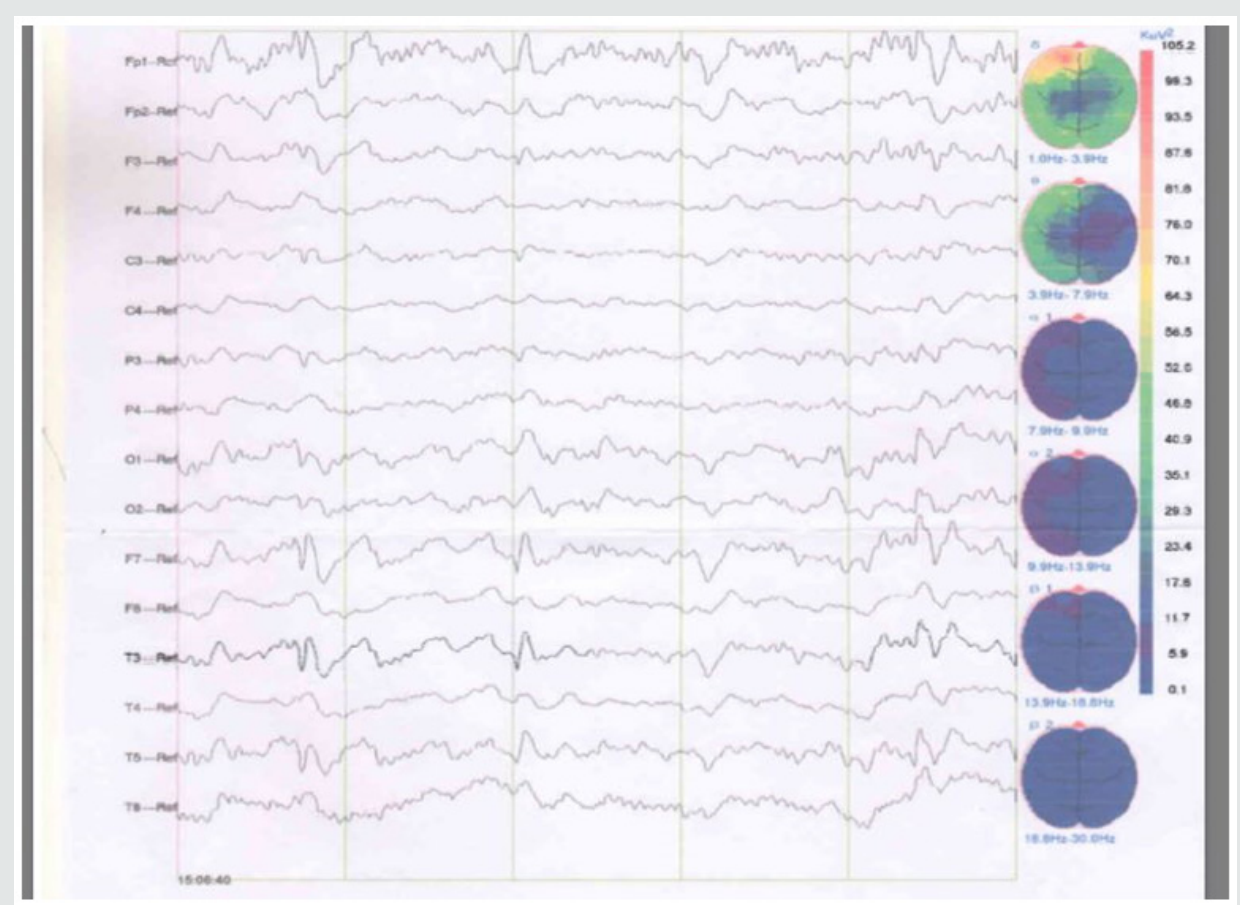

Figure 1A: Before drugs were prescribed, the patient's Electroencephalography (EEG) showed slow waves (single edge state) with no obvious epileptiform abnormalities.

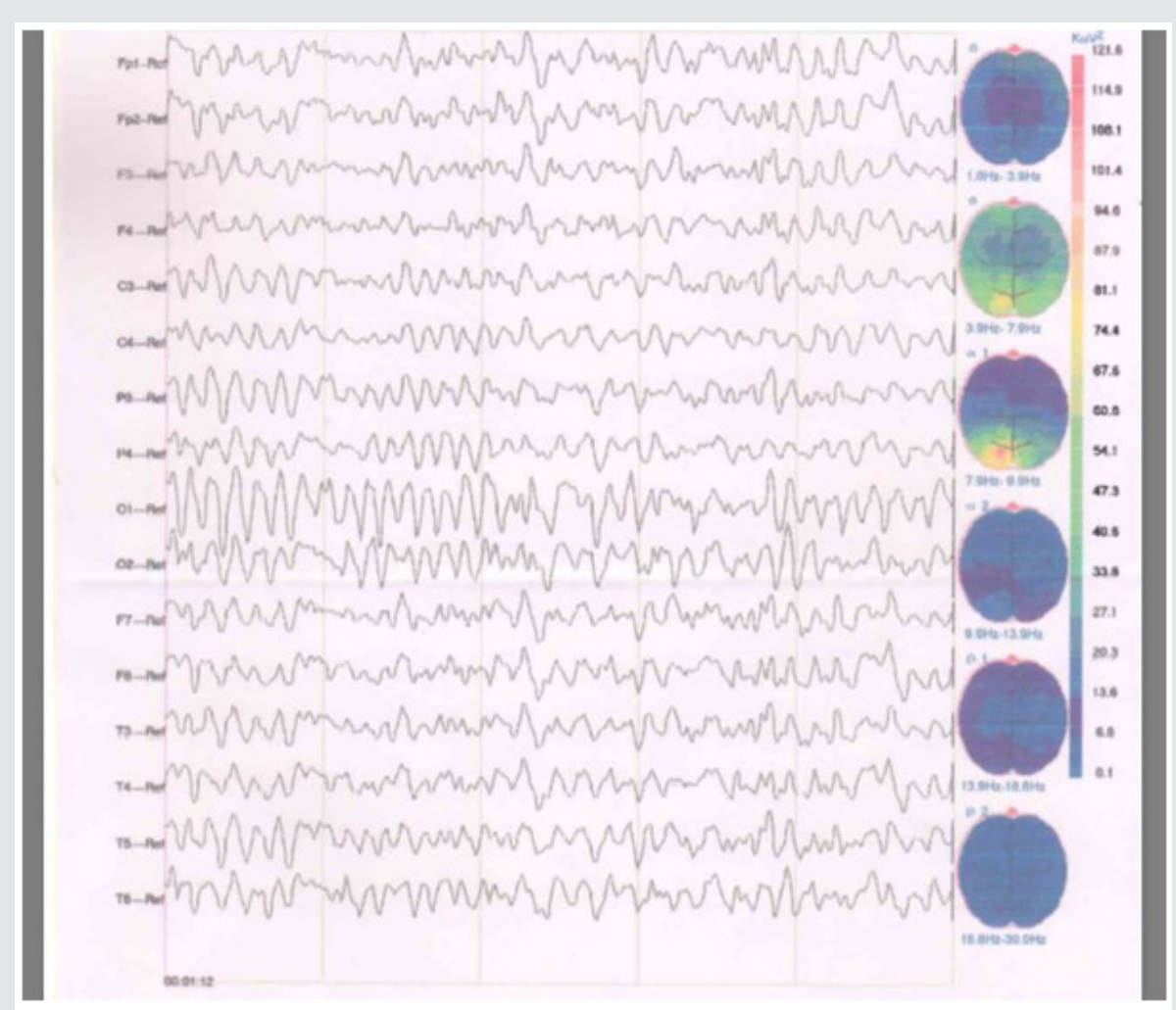

Figure 1B: In the most recent follow-up study, the patient's Electroencephalography (EEG) showed normal waves (single edge state) with no obvious epileptiform abnormalities 
In order to identify the causes of her disease, we conducted a Whole Exome Sequencing (WES) based on Next-generation sequencing. A heterozygous nonsense mutation c.1806C $>\mathrm{G}$ (p. Tyr602Ter) in TSC2 gene (reference transcript, NM_000548) was discovered in the patient. This mutation rarely occurs among either Chinese or any other ethnic groups, as it was not reported in mutation frequency databases including 1000 Genomes Database and genome AD database. This variant was further confirmed by Sanger Sequencing and was not detected in either of her healthy parents or her healthy younger brother, which indicated that this variant was a de novo mutation (Figure 2). This variant was evaluated as pathogenic mutation based on the standards and guidelines of ACMG. So far, this has been the first report of the mutation in Chinese population.
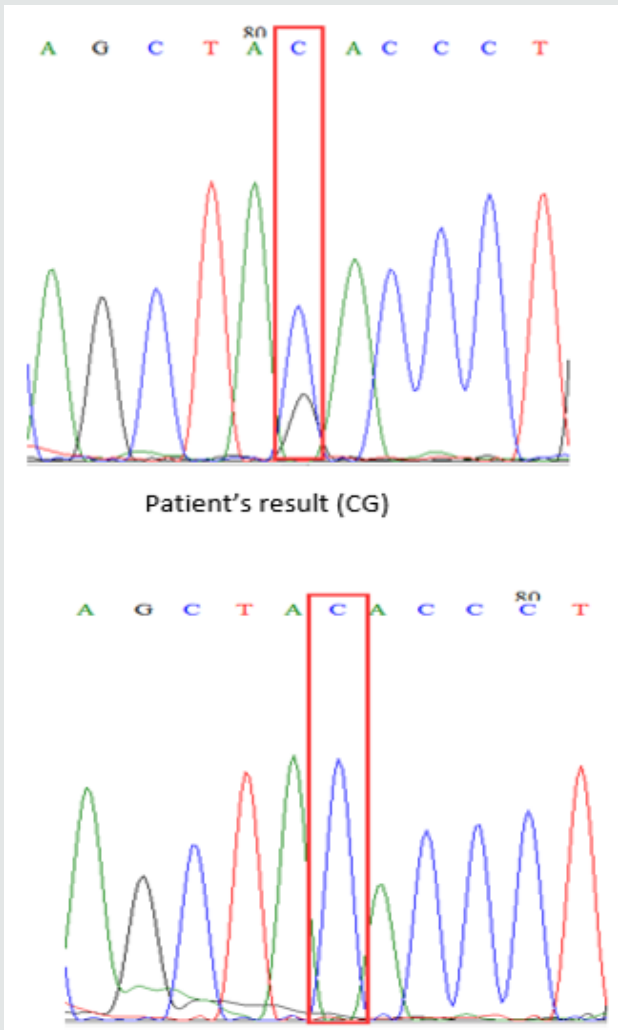

Patient's mother's result (CC)

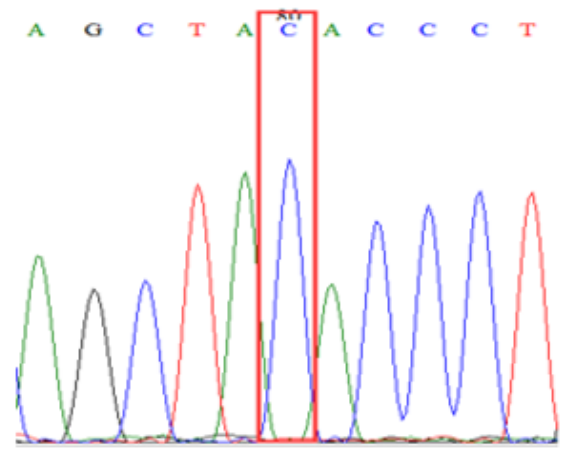

Patient's father's result (CC)

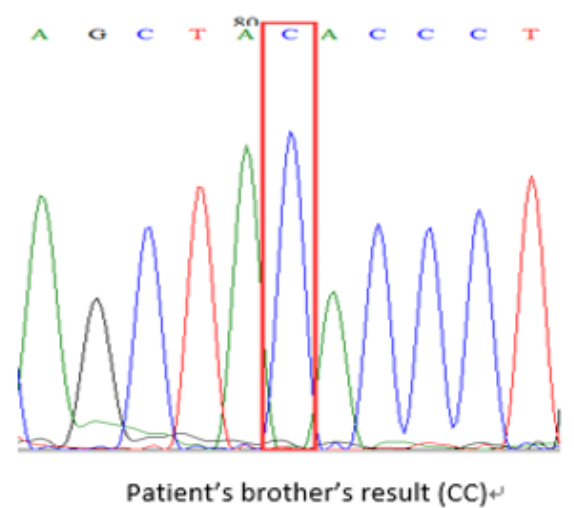

Figure 2: Sanger sequencing of the TSC2 gene mutation (c.1806C >G, p. Tyr602Ter). A: sequencing result of the patient showed a heterozygous C-to-G de novo transition (red rectangle) at codon 1806 resulting in amino acid exchange (p. Tyr602Ter). B, C, and D: Wild-type sequence (CC) in patient's father, mother and brother (red rectangles).

\section{Discussion}

In this case, we evaluated WES data from 253 Chinese patients with epilepsy and identified a de-nove heterozygous nonsense mutation in TSC2 gene (c.1806C>G (p. Tyr602Ter) in a 21-yearold female patient with Tuberous sclerosis complex (TSC). Sanger Sequencing further confirmed that it is a de novo mutation since no mutations were detected in either of her healthy parents or younger brother. This variant was evaluated as pathogenic mutation based on the standards and guidelines of ACMG.

The mutation of the gene TSC2 we detected (c.1806C $>$ G (p. Tyr602Ter) has been identified as a pathogenic mutation in Clinvar database based on the following two hypotheses. First, the Y602X variant is not observed in large population cohorts. Second, the mutation causes the loss of normal protein function through either protein truncation or nonsense-mediated mRNA decay pathway [4].
However, as of today, no detailed clinical study data or published research articles are found to support these hypotheses. Our study provided some evidence for the cause of TSC from a genetic level, and it is likely to be the first report of this mutation (p. Tyr602Ter) in Chinese population. Furthermore, our identification of this denove mutation in TSC2 gene might be helpful for advancing our understanding the role of TSC2 in TSC. The TSC2 mutations are usually detected in random cases with a frequency ranging from $75 \%$ to $80 \%$. About $15 \%$ to $20 \%$ patients do not have identifiable mutations, which may due to mosaicism $[1,2]$.

\section{Conclusion}

A novel nonsense mutation in TSC2 gene (c.1806C $>$ G (p. Tyr602Ter) was identified in a 21-year-old Chinese female patient with Tuberous sclerosis complex (TSC). This is the first report in Chinese population, which may enrich the understanding of the causes of TSC. 


\section{Consent}

Written consent was obtained from the parents of our patient for the purpose of publication of this case report and the related images. A copy of the written consent is available for review by the Editor-in-Chief of this journal.

\section{Acknowledgments}

We are grateful for the patient and her parents for their generous participation in this study. The authors have stated that they had no interests which might be perceived as conflict or bias.

\section{References}

1. Crino PB, Nathanson KL, Henske EP (2006) The tuberous sclerosis complex. N Eng J Med 355(13):1345-1356.

2. Curatolo PP, Bombardieri R, Jozwiak S (2008) Tuberous sclerosis. The Lancet 372(9639):657-668.

3. Gao X (2001) Tsc1 and tsc2 tumor suppressors antagonize insulin signaling in cell growth. Genes \& Development 15(11):1383-1392.

4. Clinvar rs367553206
(C) (1) This work is licensed under Creative

To Submit Your Article Click Here:

Submit Article

DOI: $10.32474 /$ LOJPCR.2020.02.000130

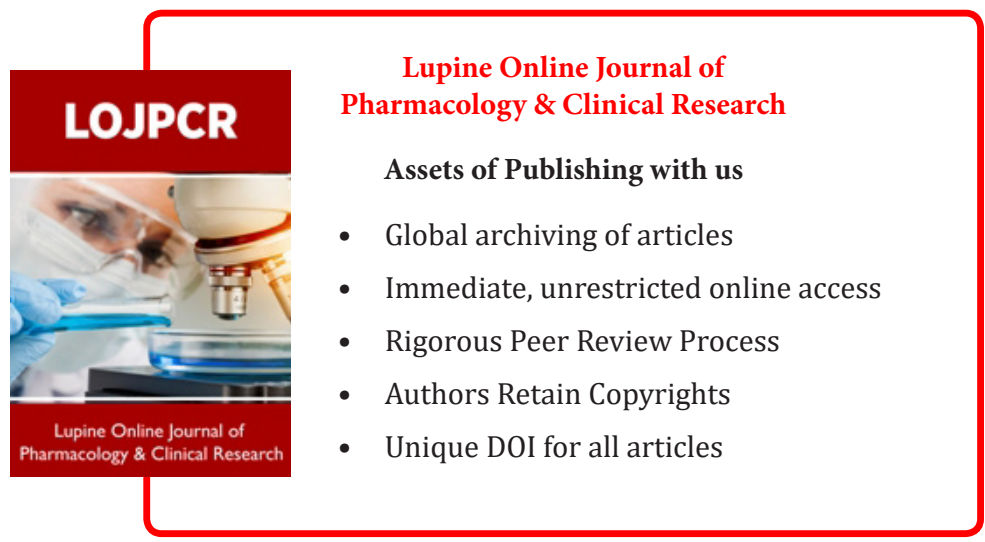

